\title{
ARTICLE Simvastatin improves olanzapine-induced dyslipidemia in rats through inhibiting hepatic mTOR signaling pathway
}

\author{
Xue-mei Liu ${ }^{1,2}$, Xiao-min Zhao ${ }^{1}$, Chao Deng ${ }^{3,4}$, Yan-ping Zeng ${ }^{1}$ and Chang-hua $\mathrm{Hu}^{1,2}$
}

\begin{abstract}
Second-generation antipsychotic drug (SGA)-induced metabolic abnormalities, such as dyslipidemia, are a major clinical problem for antipsychotic therapy. Accumulated evidences have shown the efficacy of statins in reducing SGA-induced dyslipidemia, but the underlying mechanisms are unclear. In this study, we explored whether mTOR signaling was involved in olanzapine (OLZ)-induced dyslipidemia as well as the lipid-lowering effects of cotreatment of simvastatin (Sim) in rats. Model rats received OLZ (1.0 mg/kg, t.i.d.) for 7 weeks; from the third week a group of model rats were cotreatment of Sim ( $3.0 \mathrm{mg} / \mathrm{kg}$, t.i.d.) for 5 weeks. We found that OLZ treatment significantly increased the plasma triglyceride (TG) and total cholesterol (TC) levels, and promoted lipid accumulation in the liver, whereas cotreatment of Sim reversed OLZ-induced dyslipidemia. Hepatic mTORC1 and p-mTORC1 expression was accelerated in the OLZ treatment group, with upregulation of mRNA expression of sterol regulatory element-binding protein 1c (SREBP1c) and its target genes, whereas these alterations were ameliorated by Sim cotreatment. In HepG2 cells, rapamycin (a mTOR inhibitor) significantly reduced the OLZ-stimulated hepatocellular lipid contents and weakened the ability of Sim to lower lipids via a mechanism associated with the upregulation of SREBP1c-mediated de novo lipogenesis. Our data suggest that OLZ induces lipid accumulation in both plasma and liver, and Sim ameliorates OLZ-induced lipid metabolic dysfunction through its effects on mTOR signaling via reducing SREBP1c activation and the downregulation of gene expression involved in lipogenesis. These data provide a new insight into the prevention of metabolic side effects induced by antipsychotic drugs.
\end{abstract}

Keywords: olanzapine; dyslipidemia; simvastatin; SREBP1C; de novo fatty acid synthesis; mTOR signaling pathway

Acta Pharmacologica Sinica (2019) 40:1049-1057; https://doi.org/10.1038/s41401-019-0212-1

\section{INTRODUCTION}

Use of second-generation antipsychotic drugs (SGAs), including olanzapine (OLZ), has increased exponentially worldwide for the treatment of a variety of mental disorders [1-3]. SGA-induced metabolic abnormalities, such as dyslipidemia, are a major clinical problem for antipsychotic therapy.

Over past decades, studies of the metabolic side effects induced by OLZ have focused on central nervous system (CNS) mechanisms [4]. However, previous studies have shown that OLZ can stimulate sterol regulatory element-binding protein 1c (SREBP1c) activation and expression of its downstream fatty acid synthaseregulated genes in cultured cells and the rat liver [5-8]. SREBP1c is a key transcription factor that mediates lipogenic gene expression in the liver. Recently, the mammalian target of rapamycin (mTOR) was reported to be involved in the lipid metabolic disorders induced by OLZ [9]. mTOR senses growth factors, nutrients, and cellular energy, and greatly contributes to the regulation of metabolic homeostasis [10-12]. In the liver, mTOR complex 1 (mTORC1) is thought to function as a growth factor sensor that promotes anabolic metabolism by driving protein and lipid biosynthesis [13]. Overactivation of mTORC1 may contribute to obesity and diabetes [10]. Moreover, the mTORC1 signaling pathway was reported to play an important role in regulating lipid biosynthesis and lipogenesis through SREBP1C and peroxisome proliferator-activated receptor gamma (PPAR- $\gamma$ ) [14, 15]. However, the underlying mechanisms of OLZ and whether SREBP1c is regulated through mTORC1 to stimulate de novo lipogenesis in the liver are not fully understood.

Statins are inhibitors of 3-hydroxy-3-methylglutaryl-COA (HMG$\mathrm{CoA}$ ) reductase and may be considered as a potential primary preventive and therapeutic approach for reducing SGA-induced dyslipidemia in schizophrenic patients. Many studies have reported the effect of statins, including atorvastatin [16], lovastatin [17], rosuvastatin [18], and simvastatin [19], in lowering the triglyceride (TG), total cholesterol (TC), and low-density lipoprotein cholesterol (LDL-C) levels of psychiatric patients with dyslipidemia induced by SGAs with effectiveness similar to that seen in other clinical trials. However, whether the antidyslipidemic activity of statins occurs simply through their action as a HMG-CoA reductase inhibitor or through other mechanisms is unclear. Recently, a clerodane diterpene, a new structural class of HMG-CoA reductase inhibitors apart from statins, isolated from Polyalthia longifolia has been reported to reduce lipid accumulation in preadipocytes by lowering Akt/mTOR phosphorylation [20]. Commonly, simvastatin

\footnotetext{
${ }^{1}$ School of Pharmaceutical Sciences, Southwest University, Chongqing 400715, China; ${ }^{2}$ Engineer Research Center of Chongqing Pharmaceutical Process and Quality Control, Chongqing 400715, China; ${ }^{3}$ School of Medicine, University of Wollongong, Wollongong 2522 NSW, Australia and ${ }^{4}$ Antipsychotic Research Laboratory, Illawarra Health and Medical Research Institute, Wollongong 2522 NSW, Australia

Correspondence: Chang-hua Hu (chhhu@swu.edu.cn)

These authors contributed equally: Xue-mei Liu, Xiao-min Zhao
}

Received: 29 September 2018 Accepted: 7 January 2019

Published online: 6 February 2019 
(Sim) is used as a hypolipidemic drug to control blood cholesterol levels and high cholesterol and prevent cardiovascular diseases [21]. Therefore, the purpose of this study was to evaluate the role of mTOR signaling in the dyslipidemia induced by OLZ and the lipid-lowering effects of Sim cotreatment in a rat model.

\section{MATERIALS AND METHODS}

\section{Ethical statement}

All animal experiments were performed in accordance with the National Institute of Health Guide for the Care and Use of Laboratory Animals (Publication No. 85-23, revised 1985) and were approved by the Experimental Animal Ethics Committee of the School of Pharmaceutical Sciences, Southwest University, Chongqing, China. Minimizing the number of animals and their suffering was our general practice throughout this study. For example, after the rats arrived in the animal house, they were kept for over a week without any experiments to reduce any stress caused during transportation. The rats were also handled (touched) daily during feeding to allow the animals to adapt to human contact.

Animals and treatment

Female Sprague-Dawley (SD) (45-55 g) rats were purchased from Chongqing Traditional Chinese Medicine Research Institute (China) and housed in conventional rat cages in an airconditioned room with a $12 \mathrm{~h}$ light/dark cycle (lights on: 07:00 AM, $22^{\circ} \mathrm{C}$ ) with ad libitum access to a standard laboratory chow diet and water throughout the study. The rats were trained to selfadminister a blank sweet cookie dough pellet $(0.3 \mathrm{~g}$, including $31.0 \%$ sucrose, $30.8 \%$ cornstarch, $15.5 \%$ casein, $8.4 \%$ minerals, $6.4 \%$ fiber, $6.3 \%$ gelatin, and $1.6 \%$ vitamins) for 1 week [22]. All animal experiments were performed in accordance with the National Institute of Health Guide for the Care and Use of Laboratory Animals (Publication No. 85-23, revised 1985) and were approved by the Experimental Animal Ethics Committee of School of Pharmaceutical Sciences, Southwest University, Chongqing, China.

As shown in Fig. 1, the administration plan was established as follows. The rats were randomly divided into the vehicle administration group $(n=18)$ (received a blank sweet cookie dough pellet) and the OLZ treatment group with oral administration of a pill via a cookie dough pellet $(1.0 \mathrm{mg} / \mathrm{kg}$, t.i.d, $n=18$; Eli Lilly, USA). After 2 weeks of continuous treatment, the rats in the vehicle administration group were divided into a control group ( $n$ $=9$ ) and a Sim-only group with oral administration of a pill via a cookie dough pellet $(3.0 \mathrm{mg} / \mathrm{kg}$, t.i.d, $n=9$; Merck, USA). The rats receiving OLZ treatment were divided into an OLZ-only group (1.0 $\mathrm{mg} / \mathrm{kg}$, t.i.d, $n=9)$ and an OLZ + Sim cotreatment (OLZ + Sim) group $(n=9)$ for another 5 weeks of treatment.
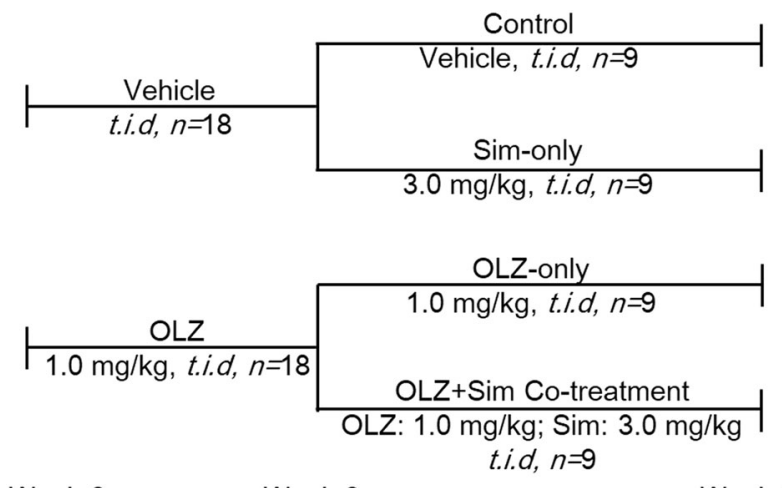

Week 0 Week $3 \quad$ Week 7

Fig. 1 Outline of the experimental design in rats
Blood and tissue sampling

After completion of drug administration, all rats were fasted for $10 \pm 2 \mathrm{~h}$ before sacrifice by ether anesthesia. The liver was collected, frozen in liquid nitrogen immediately and then stored in a $-80^{\circ} \mathrm{C}$ freezer for subsequent analysis. Cardiac blood samples were collected in EDTA-coated tubes and then centrifuged at $4{ }^{\circ} \mathrm{C}$ at $1000 \times g$ for $15 \mathrm{~min}$ to isolate the plasma. The plasma TG and TC levels were measured immediately according to the kit procedures.

Plasma lipid levels and liver enzyme measurements

Plasma TG, TC, and liver enzymes from the rats were measured using Test Kits (Nanjing JianCheng, China). After calibration with the blank tube, the absorbance of a standard tube and each sample tube was measured at 549 or $340 \mathrm{~nm}$. The concentrations were calculated according to the formula: $\left(A_{\text {test }} / A_{\text {standard }}\right) \times C_{\text {standard }}$.

Histological investigation

For detection of lipids in liver tissue, $10 \mu \mathrm{m}$ frozen sections were fixed with $10 \%$ paraformaldehyde and stained with hematoxylin and eosin (H\&E; Sigma, USA). Briefly, the slides were placed in xylene for $1 \mathrm{~min}$, followed by 100, 95, 80, and $70 \%$ ethanol for $1 \mathrm{~min}$ each. Then, hematoxylin staining was performed for $5 \mathrm{~min}$, and the slides were placed in eosin solution for $2 \mathrm{~min}$. The dehydration procedures were performed as follows: after the slides were dipped in $\mathrm{dH}_{2} \mathrm{O}$, they were incubated in $70,80,95$, and $100 \%$ ethanol for $30 \mathrm{~s}$ or $1 \mathrm{~min}$. HepG2 cells fixed in wells were stained with filtered oil red $\mathrm{O}$ solution $(0.5 \%$ in isopropanol, followed by a $60 \%$ dilution in distilled water) for $10 \mathrm{~min}$. After washing with distilled water, the slides were counterstained with hematoxylin for $2 \mathrm{~min}$, rinsed with water and mounted. For quantitative analysis of H\&E staining, two adjacent sections on the same slide ( $n=6$ for individual livers) in each treatment group were examined, and images were taken with the Olympus IX-71 microscope and quantitatively analyzed using Fiji ImageJ (https://imagej.net/Fiji).

Hepatocytes and treatment

HepG2 cells, which are a human hepatoma cell line, were obtained from the Cell Bank of the Institute of Biochemistry and Cell Biology (Shanghai, China). The cells were cultured in Dulbecco's modified Eagle's medium with $10 \%$ fetal bovine serum, $1 \mathrm{U} / \mathrm{mL}$ of penicillin and $1 \mathrm{mg} / \mathrm{mL}$ of streptomycin and were cultured in an atmosphere containing $5 \% \mathrm{CO}_{2}$ at $37^{\circ} \mathrm{C}$. All drugs were dissolved in pure DMSO (Sigma). Cell proliferation was assessed with the MTT (3-[4,5-dimethylthiazole-2-yl]-2,5-diphenyltetrazolium bromide) method in the presence of final concentrations of $10 \mu \mathrm{M} \mathrm{OLZ,}$ $25 \mu \mathrm{M}$ Sim, and $50 \mathrm{nM}$ rapamycin. DMSO was adjusted to $0.1 \%$ in the culture medium.

\section{Quantitative real-time RT-PCR}

To further explore the effects of OLZ and/or Sim cotreatment on hepatic lipid metabolism, mRNA expression was examined by quantitative RT-PCR. Total RNA was extracted from the liver tissues and cells according to the protocol of the RNA extraction kit (TianGen, China). First-strand cDNA was synthesized with the Transcriptor First Strand cDNA Synthesis Kit (Roche, Germany). Real-time PCR was performed with the SYBR Green Select Master Mix (Applied Biosystems, USA) on the MyCycler ${ }^{T M}$ system (Bio-Rad, Hercules, CA, USA). Gapdh and $\beta$-actin were used as the endogenous controls. The cycling parameters were $95^{\circ} \mathrm{C}$ for $2 \mathrm{~min}, 1 \mathrm{cycle} ; 95^{\circ} \mathrm{C}$ for $15 \mathrm{~s}, 60^{\circ} \mathrm{C}$ for $15 \mathrm{~s}$, and $72{ }^{\circ} \mathrm{C} 1 \mathrm{~min}$, 40 cycles; $65^{\circ} \mathrm{C}$ for $5 \mathrm{~s}$ and $95^{\circ} \mathrm{C}$ for $5 \mathrm{~s}$. The primer were synthesized by Shenggong (China); the sequences are shown in Table 1 (rats) and Table 2 (human). The relative mRNA levels were calculated using the formula $2^{-\Delta \Delta C t}$ and normalized to the control. 
Western blotting

For the cell line samples, the cells were washed with precooled PBS three times. RIPA lysis buffer containing 1\% $0.5 \mathrm{mM}$ PMSF were added, and the cells were incubated at $4{ }^{\circ} \mathrm{C}$ for $30 \mathrm{~min}$. After mixing with a pipette, the cells were collected and centrifuged at 12,000 rpm and $4{ }^{\circ} \mathrm{C}$ for $10 \mathrm{~min}$. For the rat liver tissues, RIPA lysis buffer containing $1 \% 0.5 \mathrm{mM}$ PMSF was added at $100 \mathrm{mg} / \mathrm{mL}$, and the tissue was fully homogenized. After centrifugation at $12,000 \mathrm{rpm}$ and $4{ }^{\circ} \mathrm{C}$ for $10 \mathrm{~min}$, the supernatant was saved as a protein extract, and the protein concentrations were quantified using the microBCA protein assay kit (Dingguo, China). The total protein extracts were separated by SDS-PAGE and transferred to a PVDF membrane. After blocking with $5 \%$ skimmed milk in TBST with $0.1 \%$ Tween 20 for $1.5 \mathrm{~h}$, the membranes were incubated with primary antibodies overnight at $4{ }^{\circ} \mathrm{C}$. The following primary antibodies were diluted 1:1000 in milk-TBS-T: SREBP1c (Abcam), LXRa/LXRß (Proteintech), $\beta$-actin (Santa Cruz), mTOR and phospho-mTOR (serine 2448) (Cell Signaling Technology). The secondary anti-mouse and antirabbit antibodies (Santa Cruz) were diluted 1:10,000 in milk-TBS-T, and the membranes were incubated with the corresponding HRP-conjugated secondary antibodies. The images with specific bands were captured with an ECL Western blotting kit according to the recommended procedure.

\begin{tabular}{|lll|}
\hline Table 1. & qRT-PCR primers (for rats) & \\
\hline Primer & Sequences $\left(5^{\prime}-3^{\prime}\right.$, forward) & Sequences $\left(5^{\prime}-3^{\prime}\right.$, reverse) \\
\hline Gapdh & ACCGCAACAGGGTGGTGGAC & TTTGAGGGTGCAGCGAACTT \\
$\beta$-Actin & CACCCGCGAGTACAACCTTC & CCCATACCCACCATCACACC \\
Srebp-1 & GGAGCCATGGATTGCACATT & CCTGTCTCACCCCCAGCATA \\
Fas & TGGTTCATTCCGTGACTG & TGTCCTTCGGTTCTTTCC \\
Acc & TGTGAACCTGAGAAATAGCA & GTGTTCAGAAGTGTGGAGC \\
Acly & ATGCCCCAAGATTCAGTCCC & TCATCTCGGGAGCACACGTA \\
Scd1 & ATGCCCCAAGATTCAGTCCC & TCATCTCGGGAGCACACGTA \\
\hline
\end{tabular}

Statistical analysis

All statistical analyses were carried out with the SPSS software (IBM version 17.0, SPSS Inc., USA), and the results were expressed as the mean $\pm \mathrm{SEM}$. Two-way $(\mathrm{OLZ} \times \mathrm{Sim})$ analysis of variance (ANOVA) was applied to analyze the data, followed by the post hoc Dunnett's $t$ test for multiple comparisons. $P<0.05$ was considered statistically significant.

\section{RESULTS}

Simvastatin decreased the fasting lipid levels in the blood and the fat accumulation in the liver induced by OLZ

As shown in Table 3, the OLZ-only treatment significantly elevated the fasting plasma TG levels (OLZ, $1.27 \pm 0.04 \mathrm{mM}$ vs. Control, $0.85 \pm$ $0.06 \mathrm{mM}, P<0.01$ ), whereas Sim-only treatment decreased the TG levels (Sim, $0.59 \pm 0.06 \mathrm{mM}$ vs. Control, $0.85 \pm 0.06 \mathrm{mM}, P<0.05$ ). The TG levels were significantly lower in the OLZ + Sim cotreatment group than in the OLZ-only group $(P<0.01)$. The plasma TC concentration was also increased in the OLZ-only group compared to that of the control (OLZ, $2.74 \pm 0.06 \mathrm{mM}$ vs. Control, $2.45 \pm 0.03$ $\mathrm{mM}, P<0.01)$, whereas Sim reduced the TC level (Sim, $2.22 \pm 0.10$ $\mathrm{mM}$ vs. Control, $2.45 \pm 0.03 \mathrm{mM}, P<0.05$ ). The TC level in the OLZ + Sim combined group was also decreased by $\sim 11.3 \%$ compared to that of the OLZ-only treatment group (OLZ + Sim, $2.43 \pm 0.05 \mathrm{mM}$ vs. OLZ, $2.74 \pm 0.06 \mathrm{mM}, P<0.01)$. Additionally, the OLZ-only rats displayed a significantly higher body weight gain $(P<0.05$, Table 3$)$ and liver weight $(P<0.05$, Fig. $2 \mathrm{~d})$, whereas the OLZ + Sim cotreatment reversed the weight gain compared with that of the OLZ-only treatment group. However, alanine aminotransferase (ALT) and aspartate transaminase (AST) showed no significant changes in any of the treatment groups.

Compared with that of the control, OLZ-only treatment significantly increased positive-ORO staining for neutral lipids $(\sim 50.0 \%$ elevation, $P<0.05)$, whereas cotreatment with Sim significantly lowered the positive ORO staining by $\sim 26.7 \%$ compared with that of the OLZ-only treatment (Fig. 2). The Sim-only group had lower lipid accumulation than the control $(\sim 10 \%$ reduction, Fig. $2 c)$.

\begin{tabular}{|lll|}
\hline Table 2. & qRT-PCR primers (for HepG2 cell line) & \\
\hline Primer & Sequences (5'-3', forward) & Sequences (5'-3', reverse) \\
\hline Gapdh & GAAGGTCGGAGTCAACGGATT & CGCTCCTGGAAGATGGTGAT \\
$\beta$-Actin & GCAAGAGAGGCATCCTCACC & CGTAGATGGGCACAGTGTGG \\
Srebp-1 & GCGGAGCCATGGATTGCAC & CTCTTCCTTGATACCAGGCCC \\
Fas & CATCGGCGACGTGGGCATTTTG & CCGGGTTCACCAGCAGGGAGCG \\
Acc & GTGGTGGTCTACTCTGATGTCA & CCACAGTGAATCTCGTTGAGA \\
Acly & ACCCAGAGGAAGCCTACATTGC & TTCGCCAGTTCGTTGACACC \\
Scd1 & CCGACGTGGCTTTTCTTCT & TGGGTGTTGCGCACAAG \\
\hline
\end{tabular}

Table 3. Plasma lipid levels and other biochemical parameters of rats after treatment of olanzapine and/or simvastatin for 5 weeks

\begin{tabular}{|c|c|c|c|c|c|c|}
\hline Control & $0.85 \pm 0.06$ & $2.45 \pm 0.03$ & $24.89 \pm 1.69$ & $66.34 \pm 6.58$ & $2.67 \pm 0.22$ & $173.3 \pm 4.5$ \\
\hline OLZ-only & $1.27 \pm 0.04^{* *}$ & $2.74 \pm 0.06^{* *}$ & $29.50 \pm 1.67$ & $75.60 \pm 2.87$ & $2.56 \pm 0.18$ & $233.2 \pm 7.9^{* *}$ \\
\hline Sim-only & $0.59 \pm 0.06^{*}$ & $2.22 \pm 0.10^{*}$ & $28.56 \pm 1.85$ & $79.21 \pm 3.74$ & $2.77 \pm 0.14$ & $153.0 \pm 6.9^{*}$ \\
\hline $\mathrm{OLZ}+\mathrm{Sim}$ & $0.84 \pm 0.03^{\# \#}$ & $2.43 \pm 0.05^{\# \#}$ & $31.23 \pm 2.11$ & $82.15 \pm 7.23$ & $2.63 \pm 0.17$ & $205.3 \pm 10.3^{\#}$ \\
\hline
\end{tabular}




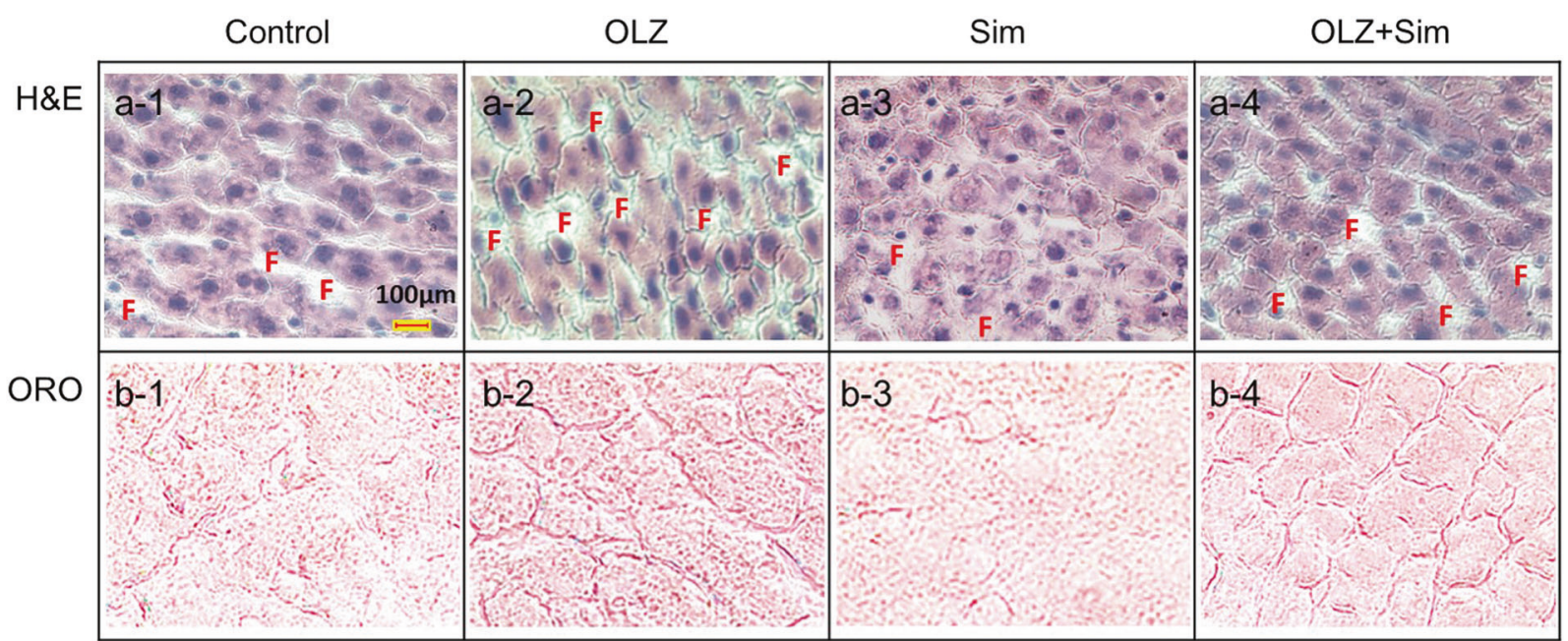

C

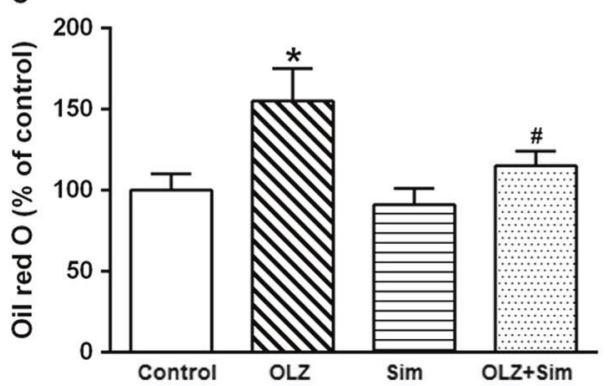

d

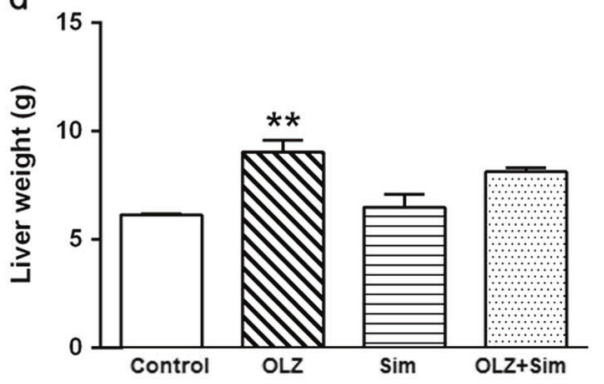

Fig. 2 Olanzapine (OLZ) treatment promoted lipid accumulation in the rat liver, whereas cotreatment with simvastatin (Sim) reduced the lipid accumulation induced by OLZ. a (1-4): Representative images counterstained with hematoxylin; b (1-4): (ORO)-stained histological sections of livers from rats treated with (a-1/b-1) the vehicle (Control), (a-2/b-2) OLZ, (a-3/b-3) Sim, (a-4/b-4), or OLZ + Sim. c Lipid levels in the liver. The ORO staining data are shown as the mean \pm SEM. d Liver weights. ORO: oil red O. F: Lipid droplets in the liver sections of the different treatment groups. ${ }^{*} P<0.05$, vs. control; ${ }^{\#} P<0.05$ vs. OLZ-only

Simvastatin treatment downregulated the expression of SREBP1 and its target genes in the rat liver

To further explore the effects of OLZ and Sim cotreatment on hepatic lipid metabolism, mRNA expression levels were examined by quantitative RT-PCR (Fig. 3), including sterol regulatory element-binding protein 1 (Srebp1), fatty acid synthase (Fasn), ATP citrate lyase (Acly), acetyl-CoA carboxylase (ACC), and stearoylCoA desaturase-1 $(S c d-1)$, as shown in Fig. 3a-e. The OLZ-only treatment significantly upregulated the expression of these genes compared to that of the control (Srebp1, 2.77 \pm 0.11 -fold, $P<0.01$; Fasn, $3.84 \pm 0.41$-fold, $P<0.01$; Acc, $1.84 \pm 0.20$-fold, $P<0.05$; Acly, $3.53 \pm 0.44$-fold, $P<0.01$, and Scd-1, $11.17 \pm 0.30$-fold, $P<0.05$ ). The Sim-only treatment significantly decreased Srebp1, Acly, and Scd-1 mRNA expression compared to that of the control $(0.43 \pm$ 0.25 -fold, $P<0.05 ; 0.47 \pm 0.40$-fold, $P<0.05$; and $0.64 \pm 0.35$-fold, $P<0.05)$. Compared to that of the OLZ-only treatment, the OLZ + Sim cotreatment resulted in a significant decrease in Srebp 1 (OLZ + Sim, $0.74 \pm 0.13$-fold vs. OLZ, $2.77 \pm 0.17$-fold, $P<0.05$ ), Fasn (OLZ + Sim, $2.00 \pm 0.37$-fold vs. OLZ, $3.85 \pm 0.41$-fold, $P<$ $0.05)$, Acc (OLZ + Sim, $0.79 \pm 0.25$-fold vs. OLZ, $1.84 \pm 0.20$-fold, $P<0.05)$, Acly (OLZ + Sim, $1.31 \pm 0.28$-fold vs. OLZ, $3.53 \pm 0.41$-fold, $P<0.05)$, and Scd -1 (OLZ + Sim, $0.66 \pm 0.31$-fold vs. OLZ, $11.47 \pm$ 0.21 -fold, $P<0.01$ ) gene expression.

Effects of OLZ and/or simvastatin treatment on mTOR signaling in the rat liver

As shown in Fig. 4, OLZ-only treatment significantly increased the mTOR total protein level $(1.26 \pm 0.10$-fold of the control, $P<0.05$;
Fig. 4b) and Ser248 phosphorylation (p-mTOR) (1.20 \pm 0.09 -fold of the control, $P<0.05$; Fig. $4 c$ ) compared to those of the control. In the Sim-only group, the relative mTOR protein expression level was $0.91 \pm 0.11$-fold that of the control, but the difference was not significant. Relative p-mTOR protein expression in the Sim-only group was $0.84 \pm 0.10$-fold that of the control $(P<0.05$; Fig. $4 \mathrm{C})$. Compared to those of the OLZ-only treatment, the OLZ and Sim cotreatment lowered the mTOR protein level by $26.19 \%(P<0.01$; Fig. $4 \mathrm{~b})$ and relative $\mathrm{p}-\mathrm{mTOR}$ expression by $17.74 \%(P<0.01$; Fig. $4 \mathrm{C})$.

Effects of OLZ and Sim cotreatment on lipid metabolism following mTOR inhibition in hepatocytes

To confirm the effect of OLZ and/or Sim cotreatment on liver lipid metabolism via the mTOR pathway, cultured HepG2 cells were stained with ORO and hematoxylin after drug treatment. The ORO staining results showed that compared to that of the control, the OLZ-only treatment significantly increased lipid droplets by $\sim 43.9 \%$ in HepG2 cells (Fig. $5 b$ vs. Fig. 5a, $P<0.01$ ), but the Sim-only treatment did not significantly change the lipid droplets (Fig. $5 c$ vs. Fig. 5a) compared to that of the control. The cotreatment with Sim decreased the OLZ-induced increase in the lipid droplets by $\sim 16.5 \%$ in HepG 2 cells (Fig. $5 d$ vs. Fig. $5 a, P<0.01$ ) compared to that of the OLZ-only treatment group. This stimulation by OLZ was abolished by the presence of rapamycin (Fig. 5f). Interestingly, the effect of Sim was enhanced by rapamycin treatment (Fig. 5h). 
a

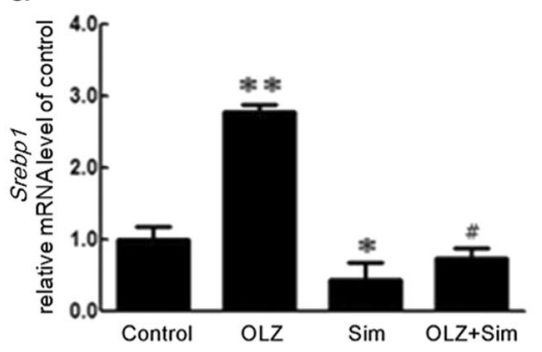

d

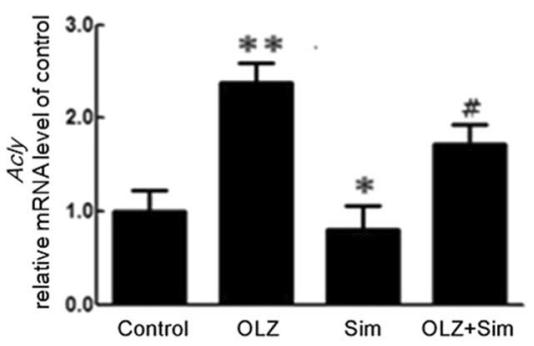

b

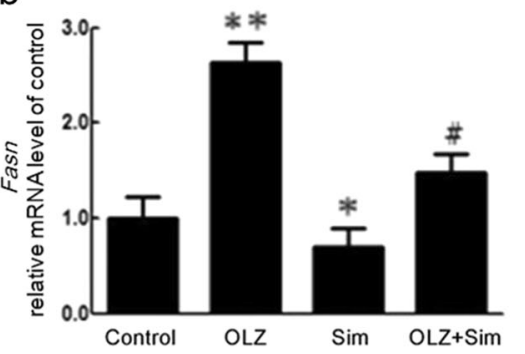

e

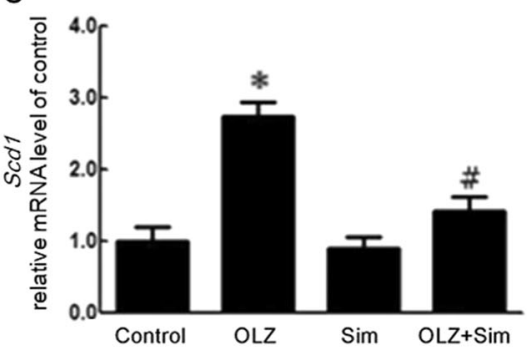

C

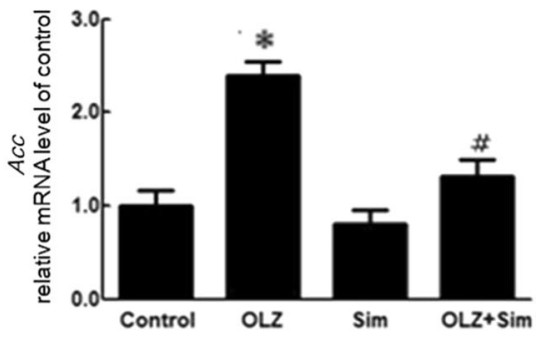

Fig. 3 Effects of olanzapine (OLZ) and/or simvastatin (Sim) cotreatment on mRNA expression of hepatic lipid synthesis-regulating genes in rats determined by quantitative RT-PCR. a Srebp-1, b Fasn, c Acc, d Acly, and e Scd1 mRNA expression. " $P<0.05$, ${ }^{* *} P<0.01$ vs. control; ${ }^{\#} P<0.05$, $\# \#<0.01$ vs. OLZ-only
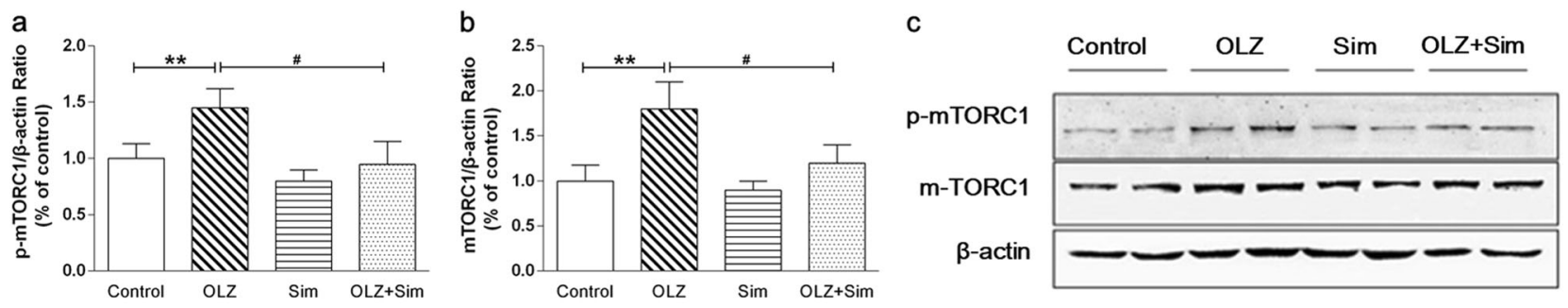

Fig. 4 Olanzapine (OLZ) treatment increased mTOR protein expression and phosphorylation in the rat liver, whereas simvastatin (Sim) reversed this upregulation. The phospho-mTOR (Ser2448) (a) and mTOR (b) protein expression levels were determined by Western blotting. c Representative immunoblots. $P<0.05$ vs. control group; ${ }^{\#} P<0.05 ;{ }^{\# \#} P<0.01$ vs. OLZ-only group
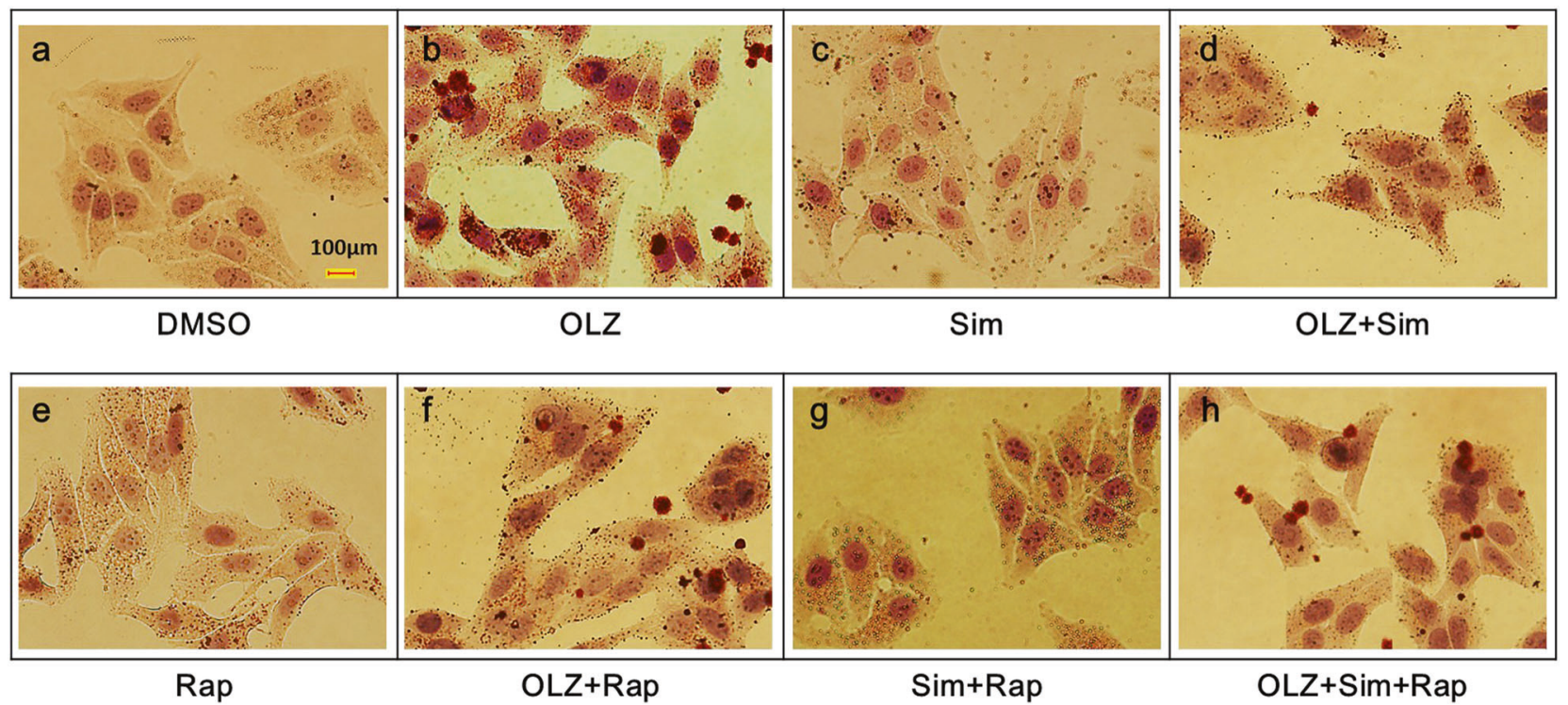

Fig. 5 Effects of olanzapine, simvastatin and rapamycin on lipid accumulation in HepG2 cells. Hepatocytes were treated with OLZ and/or Sim for $24 \mathrm{~h}$. OLZ treatment promoted lipid accumulation and Sim reduced the lipid accumulation induced by OLZ in HepG 2 cells. These effects were reversed in the presence of $50 \mathrm{nM}$ rapamycin. $\mathbf{a}-\mathbf{h}$ Representative images of HepG 2 cells stained with oil red O (ORO) after treatment with a DMSO, b $10 \mu \mathrm{M}$ of OLZ, c $25 \mu \mathrm{M}$ of Sim, d $10 \mu \mathrm{M}$ of OLZ and $25 \mu \mathrm{M}$ of Sim cotreatment, e $50 \mathrm{nM}$ of rapamycin, $\mathbf{f}$ OLZ plus rapamycin, g Sim plus rapamycin, and $\mathbf{h}$ OLZ $+\mathrm{Sim}+$ rapamycin 
a

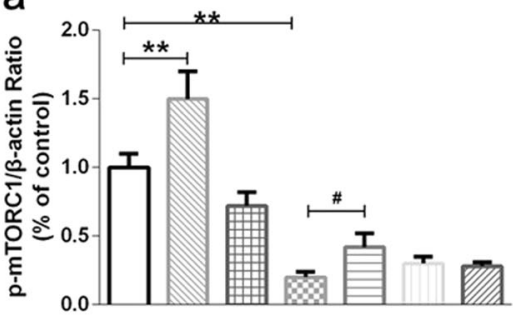

d

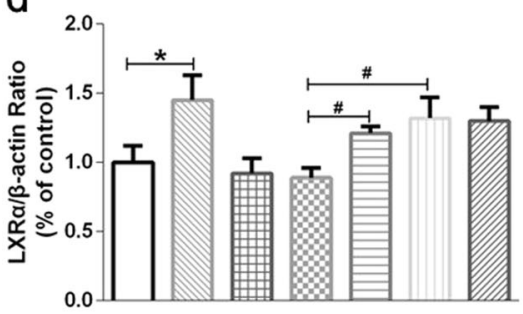

e

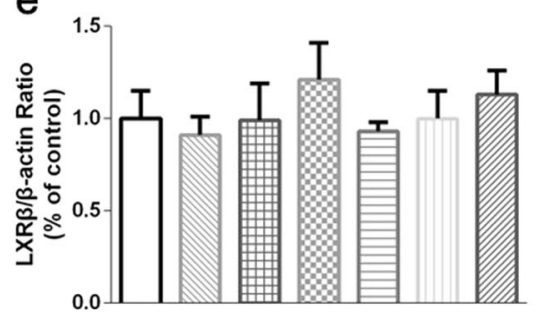

g

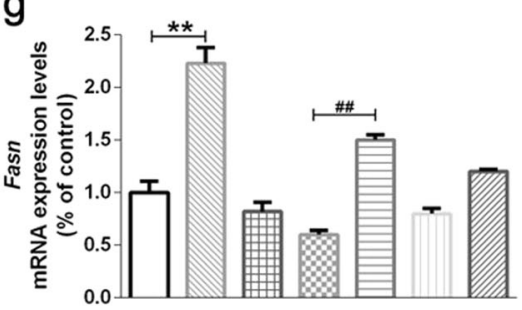

b
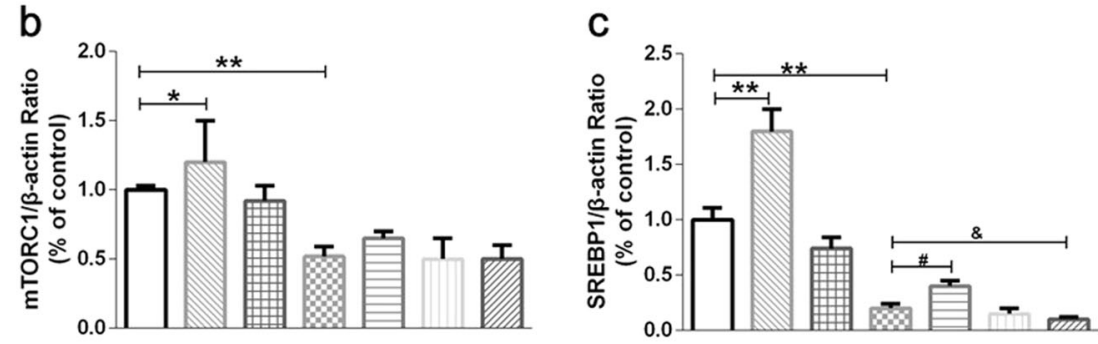

f

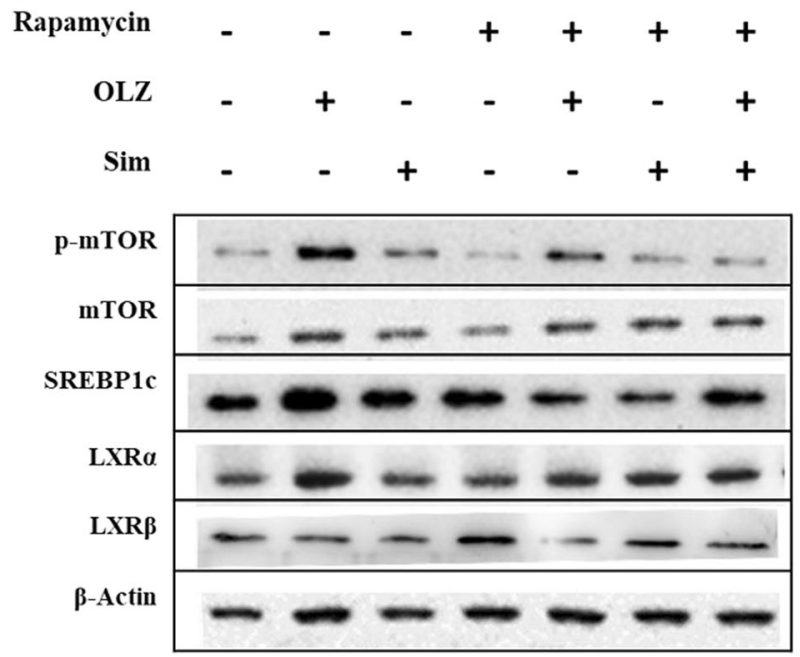

h

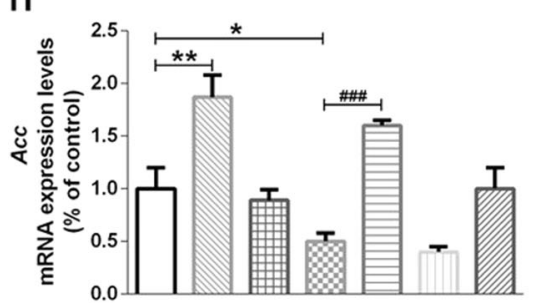

i

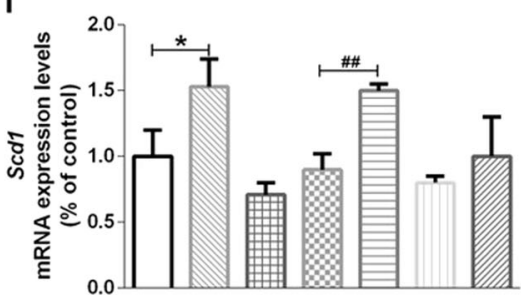

Fig. 6 The mTOR pathway is required for the inhibition of hepatic SREBP1c expression and de novo lipogenesis by simvastatin. HepG2 cells were serum-starved overnight, pretreated for $30 \mathrm{~min}$ with vehicle or rapamycin (Rap; $20 \mathrm{nM}$ ), and treated for $24 \mathrm{~h}$ with OLZ and/or Sim. The mTOR (a), p-mTOR (b), and SREBP1c protein expression levels were determined by Western blotting. $\mathbf{d}-\mathbf{f}$ mRNA expression was measured by qRT-PCR. Protein expression and transcription levels are shown relative to those of the untreated controls. g Representative immunoblots. Data are shown as the mean \pm SEM relative to the untreated control. ${ }^{*} P<0.05,{ }^{* * *} P<0.01$ vs. control group; ${ }^{\#} P<0.05,{ }^{\# \#} P<0.01$ vs. Rap group; ${ }^{\&} P<0.05,{ }^{\& \&} P<0.01$ vs. OLZ + Rap group

The mTOR pathway is required for the simvastatin-induced inhibition of hepatic SREBP1c expression and de novo lipogenesis To further define the role of $\mathrm{mTORC1}$ in the regulation of hepatic lipid metabolism by Sim, we examined the mTORC1-SREBP1c pathways in HepG2 cells. Fig. 6 shows that OLZ significantly increased $\mathrm{p}-\mathrm{mTOR}$, mTOR, SREBP1c, and LXR-a protein expression by $\sim 1.51-, \sim 1.20-, \sim 1.82-$, and $\sim 1.45$-fold, respectively (all $P<0.05$ ). The upregulation of lipogenesis gene mRNA expression, including Fasn, Acc, and Scd1, agreed with the change in the SREBP1c protein level in OLZ treatment group (Fig. $6 \mathrm{~g}-\mathrm{i}$ ). However, this study has insufficient evidence to show the reduction of these molecules upon stimulation with Sim. Pretreatment with rapamycin suppressed mTORC1 signaling (Fig. 6). Interestingly, the inhibition of $p$-mTORC1 by rapamycin was dramatically increased by OLZ (Fig. 6a, b). Moreover, OLZ administration significantly stimulated the expression of nuclear SREBP1c as well as its target genes encoding lipogenic enzymes, such as Fasn and Acc
(Fig. 6c-f). However, rapamycin significantly impaired the ability of Sim to inhibit SREBP1C activation and de novo lipid synthesis in hepatocytes. The p-mTOR, mTOR, and SREBP1c protein levels and the SREBP1c target gene mRNA level induced by OLZ were not significantly reversed by $\operatorname{Sim}$ with rapamycin pretreatment (Fig. 6a-i). Unexpectedly, pretreatment with rapamycin followed by Sim stimulation promoted LXRa protein expression $(P<0.05$; Fig. 6d).

\section{DISCUSSION}

In recent years, a variety of intervention drugs, such as metformin, berberine, betahistine, and topiramate, have been used to minimize the metabolic syndrome induced by OLZ and other SGAs $[8,23,24]$. Statins are lipid-lowering drugs that have been used for the primary prevention of cardiovascular events in patients with major mental illnesses [25]. Atorvastatin and Sim are 
the most commonly used statins, accounting for $85 \%$ of the use of these drugs [19]. We examined whether Sim treatment inhibited intracellular lipid accumulation in a hepatic cell line of human origin (HepG2) and liver tissues from OLZ-treated rats.

Numerous reports have linked SGAs, especially clozapine and OLZ, to metabolic disorders, such as dyslipidemia [26, 27]. In this study, when the rats were treated with $1.0 \mathrm{mg} / \mathrm{kg}$ (t.i.d) of OLZ for 7 weeks, the TG and TC contents in the plasma were significantly increased, which was similar to the results of previous studies [28, 29]. H\&E and ORO staining also showed that OLZ increased lipid accumulation in the liver. Moreover, $\mathrm{p}$-mTORC1 expression and the mRNA expression of SREBP1 and its target molecules (such as Acc, Fas, Acly, and Scd-1) were significantly enhanced by OLZ treatment. mTOR, which is a serine/threonine protein kinase, is found in two discrete complexes: mTOR complex 1 (mTORC1) and mTOR complex 2 (mTORC2) [30]. mTORC1 is acutely inhibited by rapamycin, which has been used therapeutically. Some studies revealed that increased mTOR activity impaired hepatocytic lipid homeostasis through regulation of transcription factor SREBP1c expression in hepatocytes [31, 32]. In the present study, inhibiting p-mTOR with rapamycin significantly reduced SREBP-1c expression, although its expression was still slightly promoted by OLZ. Taken together, our findings provide evidence that mTOR phosphorylation may be involved in OLZ-induced lipid metabolic disorder, which suggests an additional pathway other than that of the AMPactivated protein kinase (AMPK). Previously, OLZ has been found to activate hepatic AMPK signaling to mediate disturbances in glucose and lipid metabolism [9].

Statins are widely used in the treatment of patients with high serum TC and LDL-C levels [33, 34]. In schizophrenic patients treated with antipsychotics and statin therapy for 3 months, a significant decrease in the TG, TC, and LDL-C levels was observed at follow-up $[18,19,35]$. In this study, cotreatment with Sim for 5 weeks decreased the OLZ-induced improvement in the fasting plasma TG and TC levels and liver lipid accumulation. Moreover, ORO staining of HepG2 cells showed that Sim reduced lipid droplet accumulation in vitro. The general mechanism for the action of statins is their inhibitory effects on HMG-CoA reductase to lower cholesterol. In turn, SREBP1c can be activated by cytosolic cholesterol deprivation through HMG-reductase inhibition [36]. In our study, Sim ameliorated the upregulation of $\mathrm{p}$-mTORC1 caused by OLZ in the liver. Notably, pretreatment of the cells with rapamycin efficiently suppressed the Sim-mediated inhibition of SREBP1c expression, leading to increased lipogenesis and lipid accumulation in HepG2 cells. Although no studies investigating statin therapy for the dyslipidemia induced by antipsychotic drugs reported changes in the mTOR signaling pathway, we attempted to use rapamycin pretreatment to prove that the $\mathrm{mTORC} 1$ pathway was essential for the suppression of SREBP1c target gene expression by Sim. Since rapamycin could not affect HMG-CoA reductase gene expression [37], it affected the Sim-mediated inhibition of SREBP1c expression in a manner that was independent of HMG-CoA reductase $[38,39]$. Several in vitro studies have demonstrated a considerable impairment of liver $X$ receptor (LXR) signaling by statins, including reduction of the transcriptional activity of the $L X R /$ retinoid $X$ receptor heterodimer by mevastatin or lovastatin in cultured cells $[40,41]$. LXR is classified as cholesterol sensor that regulate SREBP1c gene expression, which implicates the two LXR response elements within its gene promoter [42]. Two LXR isoforms exist; the a isoform is expressed mostly in the liver, whereas the $\beta$ isoform is ubiquitously expressed. These isoforms may be downstream effectors of the mTOR signaling pathway [43]. Although Sim treatment alone did not affect LXR protein expression, pretreatment with rapamycin followed by Sim stimulation caused an increase in LXRa protein expression. From these observations, we could deduce that mTOR acted as an inhibitor of the Sim-mediated lipid-lowering responses involving LXRa. Further experiments using primary cultured hepatocytes and an LXR agonist are needed to define these effects. In addition, future studies should investigate the longterm efficacy of statins and generate more safety data for statin addon therapy in patients with schizophrenia receiving antipsychotic treatment.

OLZ and Sim are both frequently administered orally in the clinic. Since OLZ has an elimination half-life of approximately $3 \mathrm{~h}$ in rat blood [44], it was administered three times/day to maintain it at a high level in the rat brain for $8 \mathrm{~h}$. The oral dose of $1.0 \mathrm{mg} / \mathrm{kg}$ (t.i.d) of OLZ is equal to approximately $10 \mathrm{mg} /$ day in humans (60 kg body weight) according to the dosage translation between species based on body surface area following the FDA guideline $[45,46]$, which is among the recommended clinical dosages for treating schizophrenic patients [47]. Our previous studies have successfully established a female rat model for OLZ-induced metabolic side effects using oral OLZ treatment $(1.0 \mathrm{mg} / \mathrm{kg}$, t.i.d) $[29,48,49]$. Additionally, for most people with high cholesterol, the recommended starting Sim dosage is $20-40 \mathrm{mg}$ once daily $[45,46]$. Sim has been reported to have a short elimination half-life in rats of between 3.8 and $12.4 \mathrm{~h}$ after oral administration [50]. To maximize the efficacy and achieve the greatest statin concentration, $3.0 \mathrm{mg} / \mathrm{kg}$ (t.i.d) of Sim was orally administered 3 times/day to the rats in this study, which was equal to $30 \mathrm{mg}$ of drug for a patient in 1 day. Other studies have reported favorable lipid-lowing effects of simvastatin at this concentration [51, 52].

Clinically, female patients have a much higher risk than males for SGA-induced weight gain and other metabolic side effects [53-56], therefore, female rats were used in this study. Moreover, the OLZinduced weight gain model has been consistently established and validated in female rats in our and other laboratories [29, 57-59], whereas consistent modeling in male rodents has not been successful [60]. Of course, a future study in a male rat model is necessary.

In summary, OLZ induced the accumulation of hepatic lipids in both rats and hepatocyte culture, and Sim ameliorated the OLZinduced liver metabolic disorders. This reduction was partly due to its effect on mTOR pathway signaling, which reduced SREBP1c activation and contributed to downregulation of the expression of genes involved in lipogenesis. Our study provides critical information for understanding the molecular mechanisms of OLZ-associated dyslipidemia and the statin-induced lipid-lowering effect for ameliorating dyslipidemia.

\section{ACKNOWLEDGMENTS}

This work was supported by grants from the Key Program of Chongqing Science and Technology Research Project (cstc2016shmsxm80102), Venture \& Innovation Support Program for Chongqing Overseas Returnees (cx2018089) and the Fundamental Research Funds for the Central Universities, China (No. XDJK2018B037). Chao Deng was supported by a NHMRC (National Health and Medical Research Council) project grant (APP1104184).

\section{AUTHOR CONTRIBUTORS}

$\mathrm{C}-\mathrm{HH}$ and $\mathrm{X}-\mathrm{ML}$ managed the literature searches and designed the experiments. $\mathrm{X}-$ $\mathrm{MZ}$ performed the animal treatments. $\mathrm{X}-\mathrm{ML}, \mathrm{X}-\mathrm{MZ}$, and $\mathrm{Y}-\mathrm{PZ}$ performed the lipid, QPCR and Western blotting assays. X-ML and CD conducted the statistical analysis and prepared the initial draft of the manuscript. All authors contributed to and approved the final manuscript.

\section{ADDITIONAL INFORMATION}

Competing interests: The authors declare no competing interests.

\section{REFERENCES}

1. Komossa K, Depping AM, Meyer M, Kissling W, Leucht S. Second-generation antipsychotics for obsessive compulsive disorder. Cochrane Database Syst Rev. 2010;12:CD008141. 
2. Komossa K, Depping AM, Gaudchau A, Kissling W, Leucht S. Second-generation antipsychotics for major depressive disorder and dysthymia. Cochrane Database Syst Rev. 2010;12:CD008121.

3. Schneider C, Taylor D, Zalsman G, Frangou S, Kyriakopoulos M. Antipsychotics use in children and adolescents: An on-going challenge in clinical practice. J Psychopharmacol. 2014;28:615-23.

4. Weston-Green K, Huang XF, Deng C. Alterations to melanocortinergic, GABAergic and cannabinoid neurotransmission associated with olanzapine-induced weight gain. PLoS ONE. 2012;7:e33548.

5. Yang LH, Chen TM, Yu ST, Chen YH. Olanzapine induces SREBP-1-related adipogenesis in 3T3-L1 cells. Pharmacol Res. 2007;56:202-8.

6. Ferno J, Raeder MB, Vik-Mo AO, Skrede S, Glambek M, Tronstad KJ, et al. Antipsychotic drugs activate SREBP-regulated expression of lipid biosynthetic genes in cultured human glioma cells: a novel mechanism of action? Pharm J. 2005;5:298-304.

7. Raeder MB, Ferno J, Vik-Mo AO, Steen VM. SREBP activation by antipsychotic- and antidepressant-drugs in cultured human liver cells: relevance for metabolic sideeffects? Mol Cell Biochem. 2006;289:167-73.

8. Liu X, Lian J, Hu CH, Deng C. Betahistine co-treatment ameliorates dyslipidemia induced by chronic olanzapine treatment in rats through modulation of hepatic AMPKalpha-SREBP-1 and PPARalpha-dependent pathways. Pharmacol Res. 2015;100:36-46.

9. Schmidt RH, Jokinen JD, Massey VL, Falkner KC, Shi X, Yin X, et al. Olanzapine activates hepatic mammalian target of rapamycin: new mechanistic insight into metabolic dysregulation with atypical antipsychotic drugs. J Pharmacol Exp Ther. 2013;347:126-35.

10. Laplante M, Sabatini DM. mTOR signaling in growth control and disease. Cell. 2012;149:274-93.

11. Cornu M, Albert V, Hall MN. mTOR in aging, metabolism, and cancer. Curr Opin Genet Dev. 2013;23:53-62.

12. Dobrenel T, Caldana C, Hanson J, Robaglia C, Vincentz M, Veit B, et al. TOR signaling and nutrient sensing. Annu Rev Plant Biol. 2016;67:261-85.

13. Cornu M, Oppliger W, Albert V, Robitaille AM, Trapani F, Quagliata L, et al. Hepatic mTORC1 controls locomotor activity, body temperature, and lipid metabolism through FGF21. Proc Natl Acad Sci USA. 2014;111:11592-9.

14. Laplante $M$, Sabatini DM. An emerging role of mTOR in lipid biosynthesis. Curr Biol. 2009;19:R1046-52.

15. Peterson TR, Sengupta SS, Harris TE, Carmack AE, Kang SA, Balderas E, et al. mTOR complex 1 regulates lipin 1 localization to control the SREBP pathway. Cell. 2011;146:408-20.

16. Franco JM, Vallabhajosyula S, Griffin TJ. Quetiapine-induced hypertriglyceridaemia causing acute pancreatitis. BMJ Case Rep. 2015;2015:1-3.

17. Ghanizadeh A, Rezaee Z, Dehbozorgi S, Berk M, Akhondzadeh S. Lovastatin for the adjunctive treatment of schizophrenia: a preliminary randomized doubleblind placebo-controlled trial. Psychiatry Res. 2014;219:431-5.

18. De Hert M, Kalnicka D, van Winkel R, Wampers M, Hanssens L, Van Eyck D, et al. Treatment with rosuvastatin for severe dyslipidemia in patients with schizophrenia and schizoaffective disorder. J Clin Psychiatry. 2006;67:1889-96.

19. Ojala K, Repo-Tiihonen E, Tiihonen J, Niskanen L. Statins are effective in treating dyslipidemia among psychiatric patients using second-generation antipsychotic agents. J Psychopharmacol. 2008;22:33-8.

20. Beg M, Shankar K, Varshney S, Rajan S, Singh SP, Jagdale $P$, et al. A clerodane diterpene inhibit adipogenesis by cell cycle arrest and ameliorate obesity in C57BL/6 mice. Mol Cell Endocrinol. 2015;399:373-85.

21. Li J, Sun YM, Wang LF, Li ZQ, Pan W, Cao HY. Comparison of effects of simvastatin versus atorvastatin on oxidative stress in patients with coronary heart disease. Clin Cardiol. 2010;33:222-7.

22. Lian J, Huang XF, Pai N, Deng C. Ameliorating antipsychotic-induced weight gain by betahistine: mechanisms and clinical implications. Pharmacol Res. 2016; 106:51-63.

23. Maayan L, Vakhrusheva J, Correll CU. Effectiveness of medications used to attenuate antipsychotic-related weight gain and metabolic abnormalities: a systematic review and meta-analysis. Neuropsychopharmacology. 2010;35:1520-30.

24. Baptista T, ElFakih Y, Uzcategui E, Sandia I, Talamo E, de Baptista EA, et al. Pharmacological management of atypical antipsychotic-induced weight gain. CNS Drugs. 2008;22:477-95.

25. Andrade C. Primary prevention of cardiovascular events in patients with major mental illness: a possible role for statins. Bipolar Disord. 2013;15:813-23.

26. Bou Khalil R, Rohayem J, Abou Said N, El Chammay R, Haddad R, Richa S. Metabolic syndrome (MetS) in Lebanese patients with schizophrenia receiving atypical antipsychotic drugs. Asian J Psychiatr. 2013;6:88-9.

27. Said MA, Hatim A, Habil MH, Zafidah W, Haslina MY, Badiah Y, et al. Metabolic syndrome and antipsychotic monotherapy treatment among schizophrenia patients in Malaysia. Prev Med. 2013;57(Suppl):S50-3.
28. Liu X, Wu Z, Lian J, Hu CH, Huang XF, Deng C. Time-dependent changes and potential mechanisms of glucose-lipid metabolic disorders associated with chronic clozapine or olanzapine treatment in rats. Sci Rep. 2017;2764:1-7.

29. Weston-Green K, Huang XF, Deng C. Olanzapine treatment and metabolic dysfunction: a dose response study in female Sprague Dawley rats. Behav Brain Res. 2011;217:337-46.

30. Kennedy BK, Lamming DW. The mechanistic target of rapamycin: the grand ConducTOR of metabolism and aging. Cell Metab. 2016;23:990-1003.

31. Hao J, Zhu L, Li F, Liu Q, Zhao X, Liu S, et al. Phospho-mTOR: a novel target in regulation of renal lipid metabolism abnormality of diabetes. Exp Cell Res. 2013;319:2296-306.

32. Takashima M, Ogawa W, Emi A, Kasuga M. Regulation of SREBP1c expression by mTOR signaling in hepatocytes. Kobe J Med Sci. 2009;55:E45-52.

33. Sirtori CR. The pharmacology of statins. Pharmacol Res. 2014;88:3-11.

34. Mihos CG, Pineda AM, Santana O. Cardiovascular effects of statins, beyond lipidlowering properties. Pharmacol Res. 2014;88:12-9.

35. Vincenzi B, Borba CP, Gray DA, Copeland PM, Wang X, Fan X, et al. An exploratory study examining lipid-lowering medications in reducing fasting serum lipids in schizophrenia patients treated with atypical antipsychotics. Ann Clin Psychiatry. 2013;25:141-8.

36. Erol A. The role of fat tissue in the cholesterol lowering and the pleiotropic effects of statins-statins activate the generation of metabolically more capable adipocytes. Med Hypotheses. 2005;64:69-73.

37. Sharpe LJ, Brown AJ. Rapamycin down-regulates LDL-receptor expression independently of SREBP-2. Biochem Biophys Res Commun. 2008;373:670-4.

38. Torres-Ayuso P, Tello-Lafoz M, Merida I, Avila-Flores A. Diacylglycerol kinase-zeta regulates $\mathrm{mTORC1}$ and lipogenic metabolism in cancer cells through SREBP-1. Oncogenesis. 2015;4:e164.

39. Liu HW, Wei CC, Chen YJ, Chen YA, Chang SJ. Flavanol-rich lychee fruit extract alleviates diet-induced insulin resistance via suppressing mTOR/SREBP-1 mediated lipogenesis in liver and restoring insulin signaling in skeletal muscle. Mol Nutr Food Res. 2016;60:2288-96.

40. Beltowski J. Liver $X$ receptors $(\mathrm{LXR})$ as therapeutic targets in dyslipidemia. Cardiovasc Ther. 2008;26:297-316.

41. Forman BM, Ruan B, Chen J, Schroepfer GJ Jr., Evans RM. The orphan nuclear receptor LXRalpha is positively and negatively regulated by distinct products of mevalonate metabolism. Proc Natl Acad Sci USA. 1997:94:10588-93.

42. Repa JJ, Mangelsdorf DJ. The role of orphan nuclear receptors in the regulation of cholesterol homeostasis. Annu Rev Cell Dev Biol. 2000;16:459-81.

43. Zhou Y, Yu S, Cai C, Zhong L, Yu H, Shen W. LXRa participates in the mTOR/S6K1/ SREBP-1C signaling pathway during sodium palmitate-induced lipogenesis in HepG2 cells. Nutr Metab (Lond). 2018;15:31.

44. Liu X, Deng C, Cao S, Gong J, Wang BC, Hu CH. Acute effects of oral olanzapine treatment on the expression of fatty acid and cholesterol metabolism-related gene in rats. Life Sci. 2015;128:72-8.

45. Reagan-Shaw S, Nihal M, Ahmad N. Dose translation from animal to human studies revisited. FASEB J. 2008;22:659-61.

46. FDA. Estimating the Maximum Safe Starting Dose in Initial Clinical Trials for Therapeutics in Adult Healthy Volunteers. http://www.fda.gov/cder/guidance/ index.htm 2005.

47. Kantrowitz JT, Citrome L. Olanzapine: review of safety 2008. Expert Opin Drug Saf. 2008;7:761-9.

48. Lian J, Huang XF, Pai N, Deng C. Betahistine ameliorates olanzapine-induced weight gain through modulation of histaminergic, NPY and AMPK pathways. Psychoneuroendocrinology. 2014;48:77-86.

49. Weston-Green K, Huang XF, Lian J, Deng C. Effects of olanzapine on muscarinic M3 receptor binding density in the brain relates to weight gain, plasma insulin and metabolic hormone levels. Eur Neuropsychopharmacol. 2012;22:364-73.

50. Xu D, Li F, Zhang M, Zhang J, Liu C, Hu MY, et al. Decreased exposure of simvastatin and simvastatin acid in a rat model of type 2 diabetes. Acta Pharmacol Sin. 2014:35:1215-25.

51. Tan J, Yang N, Fu X, Cui Y, Guo Q, Ma T, et al. Single-dose local simvastatin injection improves implant fixation via increased angiogenesis and bone formation in an ovariectomized rat model. Med Sci Monit. 2015;21:1428-39.

52. Barbosa CP, Bracht L, Ames FQ, de Souza Silva-Comar FM, Tronco RP, BersaniAmado CA. Effects of ezetimibe, simvastatin, and their combination on inflammatory parameters in a rat model of adjuvant-induced arthritis. Inflammation. 2017:40:717-24.

53. Weston-Green K, Huang XF, Deng C. Sensitivity of the female rat to olanzapineinduced weight gain-Far from the clinic? Schizophr Res. 2010;116:299-300.

54. Seeman MV. Secondary effects of antipsychotics: women at greater risk than men. Schizophr Bull. 2009;35:937-48.

55. Gebhardt S, Haberhausen M, Heinzel-Gutenbrunner M, Gebhardt N, Remschmidt $\mathrm{H}$, Krieg J-C, et al. Antipsychotic-induced body weight gain: predictors and a 
systematic categorization of the long-term weight course. J Psychiatr Res. 2009;43:620-6.

56. Treuer T, Pendlebury J, Lockman H, Bushe C, Karagianis J, Raskin J, et al. Weight Gain Risk Factor assessment checklist: overview and recommendation for use. Neuroendocrinol Lett. 2011;32:199-205.

57. Deng C, Lian J, Pai N, Huang XF. Reducing olanzapine-induced weight gain side effect by using betahistine: a study in the rat model. J Psychopharmacol. 2012;26:1271-9.
58. Choi S, DiSilvio B, Unangst J, Fernstrom JD. Effect of chronic infusion of olanzapine and clozapine on food intake and body weight gain in male and female rats. Life Sci. 2007;81:1024-30.

59. Goudie AJ, Smith J, Halford J. Characterization of olanzapine-induced weight gain in rats. J Psychopharmacol. 2002;16:291-6.

60. van der Zwaal EM, Janhunen SK, la Fleur SE, Adan RAH. Modelling olanzapine-induced weight gain in rats. Int J Neuropsychopharmacol. 2014; 17:169-86. 